\title{
Characterisation of micromechanical properties using advanced techniques
}

\author{
Manuel A. Pouchon ${ }^{1)}$, Rudy Ghisleni²), Johann Michler ${ }^{2)}$ \\ 1) Paul Scherrer Institute, 5232 Villigen PSI, Switzerland \\ 2) EMPA, Feuerwerkerstrasse 39, 3602 Thun, Switzerland
}

The mechanical testing of very small material volumes is motivated by different reasons. In some cases the micromechanical testing allows to address more fundamental properties, as simplified systems, e.g. single grains within a polycrystalline materials, can selectively be tested. In other cases especially application relevant locations within the material can be selected, as for example welds or treated locations, and characterized. An example for treated regions are ion implanted surfaces, which can be used to study radiation damage in materials [1] or simply to provide a surface finish with enhanced properties for technical applications. Subsample testing reduces the required sample volume, which is helpful if the samples are very rare and/or are difficult to handle in large volumes/quantities. [2] shows studies where radioactive materials were extensively studied using miniaturized testing techniques. Today's sophisticated sample preparation techniques, such as feature extractions using a focused ion beam (FIB), even allow the isolation of material interfaces of special interest, such as grain boundaries, and test them. Even the testing of features within composite materials is possible, as fibre push-out tests in fibre reinforced ceramics have shown [3]. In future the mechanical property characterisation of small devices gains importance, as more of these components are going to be developed. An example are microelectromechanical systems (MEMS) where due to the very small size standard methods like tensile tests can no longer be applied. Furthermore, new mechanical properties can be expected on small scales which must be understood. Therefore new characterisation techniques must be applied [4].

The paper will show examples demonstrating implementations of micromechanical testing for some of the above mentioned applications. Some of these will also show in situ visualisations helping to identify damage events, as the activation of glide planes. Figure 1 depicts a micropillars being extracted from a reactor pressure vessel steel after an irradiation, an annealing and a re-irradiation step. The figure shows the pillar before and after testing. Many more samples of this material with different irradiation and annealing histories have been tested this way and especially an in-situ SEM testing video shows the Lüders strain regime of an annealed sample.

\section{References}

[1] M.A. Pouchon, J. Chen, R. Ghisleni, J. Michler, W. Hoffelner, Exp. Mech. 50[1] (2010) 7984. (DOI:10.1007/s11340-008-9214-5)

[2] P. Hosemann, E. Stergar, L. Peng, Y. Dai, S.A. Maloy, M.A. Pouchon, K. Shiba, D. Hamaguchi, H. Leitner, , J. Nucl. Mat., 417 [1-3], (2011) 274-278

[3] A.F. Kalton, S.J. Howard, J. Janczak-Rusch, T.W. Clyne, Acta Mat., 46 [9] (1998) 3175-3189 DOI: 10.1016/S1359-6454(98)00009-3. 
[4] P. Schwaller, S. Stauss, J. Michler, G. Genolet, H. Lorenz, L. Rohr, 3rd Korea-Switzerland Joint Symposium on "New Materials and Process Issues in MEMS and Nanotechnologies, (2002) 141-149

[5] IAEA-TECDOC-1230
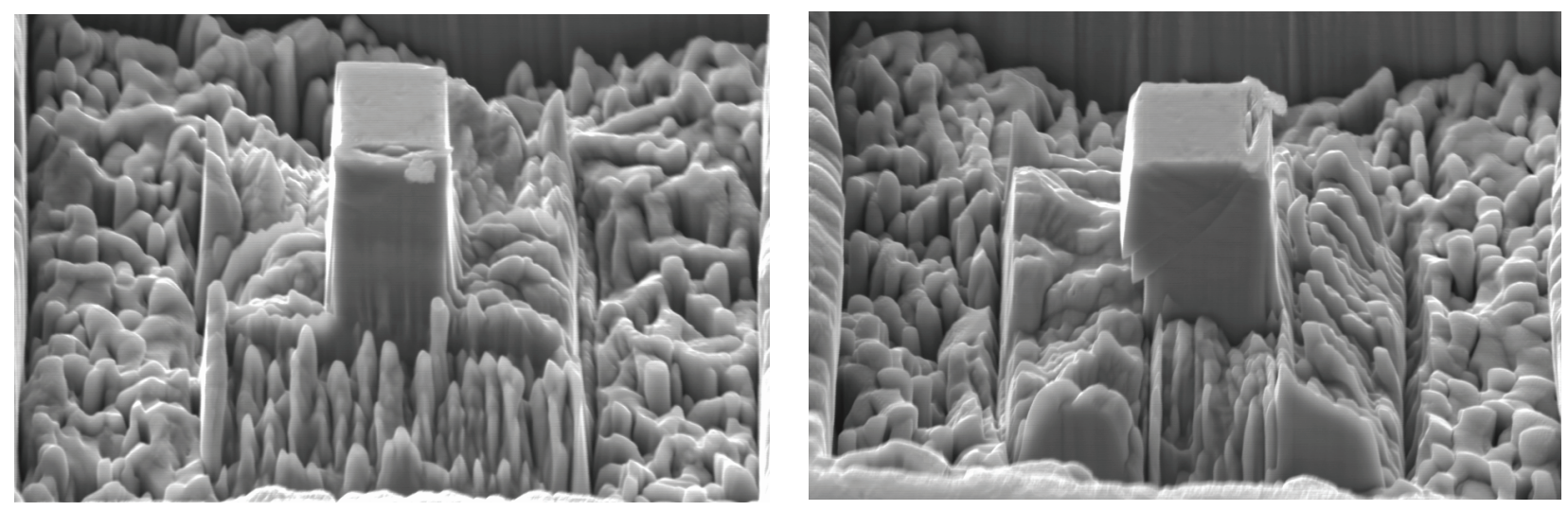

Fig. 1: Reactor pressure vessel steel: JRQ sample material [5]. $2 \times 2 \times 4 \mu^{3}$ square pillars before and after compression test. 